\title{
AUTOMORPHISM GROUPS OF ULTRAPRODUCTS OF FINITE SYMMETRIC GROUPS
}

\author{
PHILIPP LÜCKE AND SIMON THOMAS
}

\begin{abstract}
It is consistent that there exists a nonprincipal ultrafilter $\mathcal{U}$ over $\mathbb{N}$ such that every automorphism of the corresponding ultraproduct $\prod_{\mathcal{U}} \operatorname{Sym}(n)$ is inner.
\end{abstract}

\section{INTRODUCTION}

Suppose that $\mathcal{U}$ is a nonprincipal ultrafilter over $\mathbb{N}$ and that $\prod_{\mathcal{U}} \operatorname{Sym}(n)$ is the corresponding ultraproduct of the finite symmetric groups. If $C H$ holds, then $\prod_{\mathcal{U}} \operatorname{Sym}(n)$ is a saturated structure and hence

$$
\left|\operatorname{Aut}\left(\prod_{\mathcal{U}} \operatorname{Sym}(n)\right)\right|=2^{\aleph_{1}}>\aleph_{1}=\left|\prod_{\mathcal{U}} \operatorname{Sym}(n)\right| .
$$

(For example, see Chang-Keisler [1].) In particular, if $C H$ holds, then $\prod_{\mathcal{U}} \operatorname{Sym}(n)$ has many outer automorphisms. Of course, it is well-known that if $n \neq 6$, then every automorphism of $\operatorname{Sym}(n)$ is inner; and consequently, it appears to be difficult to exhibit an explicit example of an outer automorphism of $\prod_{\mathcal{U}} \operatorname{Sym}(n)$. The main result of this paper confirms that this is indeed a genuine difficulty.

Theorem 1.1. It is consistent that there exists a nonprincipal ultrafilter $\mathcal{U}$ over $\mathbb{N}$ such that every automorphism of $\prod_{\mathcal{U}} \operatorname{Sym}(n)$ is inner.

No knowledge of set theory is needed in order to understand the proof of Theorem 1.1, which is a purely algebraic consequence of the following remarkable result on ultraproducts of the fields $\mathbb{F}_{p}$ of prime order $p$. (Recall that a structure $\mathcal{M}$ is said to be rigid if the identity map is the only automorphism of $\mathcal{M}$.)

2000 Mathematics Subject Classification. 03C20, 03E35, 20B30, 20E36.

Key words and phrases. Ultraproducts, inner automorphisms.

The research of the first author was supported by DFG Grant SCHI 484/4-1.

The research of the second author was partially supported by NSF Grant DMS 0600940. 
Theorem 1.2 (Shelah [6]). It is consistent that there exists a nonprincipal ultrafilter $\mathcal{F}$ over the set $\mathbb{P}$ of primes such that the field $\prod_{\mathcal{F}} \mathbb{F}_{p}$ is rigid.

This paper is organized as follows. In Section 2, we will recall the notion of an internal automorphism of an ultraproduct; and prove that if the field $\prod_{\mathcal{F}} \mathbb{F}_{p}$ is rigid, then every automorphism of $\prod_{\mathcal{F}} P S L_{2}\left(\mathbb{F}_{p}\right)$ is internal. In Section 3, we will recall two basic results concerning regular permutation representations. Finally, in Section 4, we will present the proof of Theorem 1.1 .

\section{The GROUP OF INTERNAL AUTOMORPHISMS}

In this section, we will recall the notion of an internal automorphism of an ultraproduct; and prove that if the field $\prod_{\mathcal{F}} \mathbb{F}_{p}$ is rigid, then every automorphism of $\prod_{\mathcal{F}} P S L_{2}\left(\mathbb{F}_{p}\right)$ is internal.

Suppose that $G_{i}, i \in I$, are groups and that $\mathcal{U}$ is a nonprincipal ultrafilter over the index set $I$. Then an automorphism $\varphi \in \operatorname{Aut}\left(\prod_{\mathcal{U}} G_{i}\right)$ is said to be internal if there exist automorphisms $\varphi_{i} \in \operatorname{Aut}\left(G_{i}\right)$ such that for all $\left(x_{i}\right)_{\mathcal{U}} \in \prod_{\mathcal{U}} G_{i}$,

$$
\varphi\left(\left(x_{i}\right) \mathcal{U}\right)=\left(\varphi_{i}\left(x_{i}\right)\right) \mathcal{U}
$$

The group of internal automorphisms of $\prod_{\mathcal{U}} G_{i}$ is denoted by $\operatorname{Int}\left(\prod_{\mathcal{U}} G_{i}\right)$. Clearly we have that

$$
\operatorname{Inn}\left(\prod_{\mathcal{U}} G_{i}\right) \unlhd \operatorname{Int}\left(\prod_{\mathcal{U}} G_{i}\right) \leqslant \operatorname{Aut}\left(\prod_{\mathcal{U}} G_{i}\right)
$$

Example 2.1. Recall that if $n \neq 6$, then every automorphism of $\operatorname{Sym}(n)$ is inner. It follows that if $\mathcal{U}$ is any nonprincipal ultrafilter over $\mathbb{N}$, then

$$
\operatorname{Int}\left(\prod_{\mathcal{U}} \operatorname{Sym}(n)\right)=\operatorname{Inn}\left(\prod_{\mathcal{U}} \operatorname{Sym}(n)\right)
$$

Hence Theorem 1.1 is equivalent to the consistency of a nonprincipal ultrafilter $\mathcal{U}$ over $\mathbb{N}$ such that

$$
\operatorname{Aut}\left(\prod_{\mathcal{U}} \operatorname{Sym}(n)\right)=\operatorname{Int}\left(\prod_{\mathcal{U}} \operatorname{Sym}(n)\right)
$$

Lemma 2.2. If $\mathcal{F}$ is a nonprincipal ultrafilter over the set $\mathbb{P}$ of primes such that the field $\prod_{\mathcal{F}} \mathbb{F}_{p}$ is rigid, then

$$
\operatorname{Aut}\left(\prod_{\mathcal{F}} P S L_{2}\left(\mathbb{F}_{p}\right)\right)=\operatorname{Int}\left(\prod_{\mathcal{F}} P S L_{2}\left(\mathbb{F}_{p}\right)\right)
$$


Proof. From now on, if $K$ is any field and $\left(\begin{array}{ll}a & b \\ c & d\end{array}\right) \in S L_{2}(K)$, then $\left[\begin{array}{ll}a & b \\ c & d\end{array}\right]$ denotes the corresponding element of $P S L_{2}(K)$. Following Kegel-Wehrfritz [3, 1.L.6], we can define an isomorphism $\prod_{\mathcal{F}} P S L_{2}\left(\mathbb{F}_{p}\right) \rightarrow P S L_{2}\left(\prod_{\mathcal{F}} \mathbb{F}_{p}\right)$ by

$$
\left(\left[\begin{array}{cc}
a_{p} & b_{p} \\
c_{p} & d_{p}
\end{array}\right]\right)_{\mathcal{F}} \mapsto\left[\begin{array}{ll}
\left(a_{p}\right)_{\mathcal{F}} & \left(b_{p}\right)_{\mathcal{F}} \\
\left(c_{p}\right)_{\mathcal{F}} & \left(d_{p}\right)_{\mathcal{F}}
\end{array}\right]
$$

By a well-known theorem of Schreier and van der Waerden [4], if $K$ is any field with $|K|>3$, then every automorphism of $P S L_{2}(K)$ is induced via conjugation by an element of

$$
P \Gamma L_{2}(K)=P G L_{2}(K) \rtimes \operatorname{Aut}(K) .
$$

In particular, since the field $\prod_{\mathcal{F}} \mathbb{F}_{p}$ is rigid, every automorphism of $P S L_{2}\left(\prod_{\mathcal{F}} \mathbb{F}_{p}\right)$ is induced via conjugation by an element of $P G L_{2}\left(\prod_{\mathcal{F}} \mathbb{F}_{p}\right)$. This implies that every automorphism of $\prod_{\mathcal{F}} P S L_{2}\left(\mathbb{F}_{p}\right)$ is induced via conjugation by an element of $\prod_{\mathcal{F}} P G L_{2}\left(\mathbb{F}_{p}\right)$ and hence

$$
\operatorname{Aut}\left(\prod_{\mathcal{F}} P S L_{2}\left(\mathbb{F}_{p}\right)\right)=\operatorname{Int}\left(\prod_{\mathcal{F}} P S L_{2}\left(\mathbb{F}_{p}\right)\right)
$$

\section{Regular PeRmutation RePRESEntations}

In this section, we will recall two basic results concerning regular permutation representations. Here the left regular permutation representation of the group $H$ is the embedding $\lambda: H \rightarrow \operatorname{Sym}(H)$ defined by $\lambda(h)(x)=h x$; and the right regular permutation representation of $H$ is the embedding $\rho: H \rightarrow \operatorname{Sym}(H)$ defined by $\rho(h)=x h^{-1}$. The proof of Theorem 1.1 makes use of the following well-known result. (For example, see Hall [2, Theorem 6.3.1].)

Lemma 3.1. If $H$ is any group, then:

(a) $C_{\operatorname{Sym}(H)}(\lambda[H])=\rho[H]$; and

(b) $C_{\operatorname{Sym}(H)}(\rho[H])=\lambda[H]$.

If $H$ is any group, then the holomorph of $H$ is the subgroup $\operatorname{Hol}(H)$ of $\operatorname{Sym}(H)$ defined by

$$
\operatorname{Hol}(H)=\lambda[H] \rtimes \operatorname{Aut}(H) .
$$


It is easily checked that $\operatorname{Hol}(H)=\rho[H] \rtimes \operatorname{Aut}(H)$. The proof of Theorem 1.1 also makes use of the following result. (For example, see Hall [2, Theorem 6.3.2].)

Lemma 3.2. If $H$ is any group, then

$$
N_{\mathrm{Sym}(H)}(\lambda[H])=N_{\mathrm{Sym}(H)}(\rho[H])=\operatorname{Hol}(H) .
$$

Finally recall that if $\Omega$ is any set, then a subgroup $L$ of $\operatorname{Sym}(\Omega)$ is said to be regular if for each pair of (not necessarily distinct) elements $x, y \in \Omega$, there exists a unique element $\pi \in L$ such that $\pi(x)=y$. In this case, the permutation group ( $L, \Omega)$ is isomorphic to the left regular permutation representation of $L$ in $\operatorname{Sym}(L)$; and, of course, is also isomorphic to the right regular permutation representation.

\section{The Proof of Theorem 1.1}

In this section, we will present the proof of Theorem 1.1. Throughout, we fix a nonprincipal ultrafilter $\mathcal{F}$ over the set $\mathbb{P}$ of primes such that the field $\prod_{\mathcal{F}} \mathbb{F}_{p}$ is rigid. For each prime $p \in \mathbb{P}$, let $\Gamma_{p}=P S L_{2}\left(\mathbb{F}_{p}\right)$. Let $G_{\mathcal{F}}=\prod_{\mathcal{F}} \operatorname{Sym}\left(\Gamma_{p}\right)$ and $H_{\mathcal{F}}=\prod_{\mathcal{F}} \Gamma_{p}$. Then it is clearly enough to show that every automorphism of $G_{\mathcal{F}}$ is inner.

For each prime $p \in \mathbb{P}$, let $\lambda_{p}: \Gamma_{p} \rightarrow \operatorname{Sym}\left(\Gamma_{p}\right)$ and $\rho_{p}: \Gamma_{p} \rightarrow \operatorname{Sym}\left(\Gamma_{p}\right)$ be the left regular and right regular permutation representations. Consider the action of $G_{\mathcal{F}}$ on $H_{\mathcal{F}}$ defined by

$$
\left(\pi_{p}\right)_{\mathcal{F}} \cdot\left(\gamma_{p}\right)_{\mathcal{F}}=\left(\pi_{p}\left(\gamma_{p}\right)\right)_{\mathcal{F}}
$$

Then, identifying the group $G_{\mathcal{F}}$ with its image under the corresponding embedding $G_{\mathcal{F}} \rightarrow \operatorname{Sym}\left(H_{\mathcal{F}}\right)$, we have that $\prod_{\mathcal{F}} \lambda_{p}\left[\Gamma_{p}\right]$ corresponds to the image $\lambda\left[H_{\mathcal{F}}\right]$ of the left regular permutation representation of $H_{\mathcal{F}}$. Also notice that under this identification,

$$
\operatorname{Alt}\left(H_{\mathcal{F}}\right) \leqslant G_{\mathcal{F}} \leqslant \operatorname{Sym}\left(H_{\mathcal{F}}\right) .
$$

Hence, by Scott $[5,11.4 .7]$, it follows that $\operatorname{Aut}\left(G_{\mathcal{F}}\right)$ is precisely the normalizer of $G_{\mathcal{F}}$ in $\operatorname{Sym}\left(H_{\mathcal{F}}\right)$.

Next let $\Gamma=P S L_{2}(\mathbb{Z})$. Then it is well-known that $\Gamma$ is generated by the following two elements:

$$
a=\left[\begin{array}{ll}
1 & 1 \\
0 & 1
\end{array}\right] \quad b=\left[\begin{array}{ll}
1 & 0 \\
1 & 1
\end{array}\right]
$$


For each prime $p \in \mathbb{P}$, let $a_{p}, b_{p} \in P S L_{2}\left(\mathbb{F}_{p}\right)$ be the images of $a, b \in P S L_{2}(\mathbb{Z})$ under the canonical surjective homomorphism $P S L_{2}(\mathbb{Z}) \rightarrow P S L_{2}\left(\mathbb{F}_{p}\right)$; and define the elements $\alpha, \beta \in \prod_{\mathcal{F}} \operatorname{Sym}\left(\Gamma_{p}\right)$ by $\alpha=\left(\rho_{p}\left(a_{p}\right)\right)_{\mathcal{F}}$ and $\beta=\left(\rho_{p}\left(b_{p}\right)\right)_{\mathcal{F}}$. Applying Lemma 3.1, since $\Gamma_{p}=\left\langle a_{p}, b_{p}\right\rangle$, it follows that

$$
C_{\mathrm{Sym}\left(\Gamma_{p}\right)}\left(\rho_{p}\left(a_{p}\right), \rho_{p}\left(b_{p}\right)\right)=\lambda_{p}\left[\Gamma_{p}\right]
$$

and hence, by Łoś's Theorem [1],

$$
C_{G_{\mathcal{F}}}(\alpha, \beta)=\prod_{\mathcal{F}} \lambda_{p}\left[\Gamma_{p}\right] .
$$

Now suppose that $\pi \in \operatorname{Aut}\left(G_{\mathcal{F}}\right)$ is any automorphism. Let $\pi(\alpha)=\left(g_{p}\right)_{\mathcal{F}}$ and let $\pi(\beta)=\left(h_{p}\right)_{\mathcal{F}}$. Then

$$
C_{G_{\mathcal{F}}}\left(\left(g_{p}\right)_{\mathcal{F}},\left(h_{p}\right)_{\mathcal{F}}\right)=\prod_{\mathcal{F}} \Delta_{p},
$$

where $\Delta_{p}=C_{\operatorname{Sym}\left(\Gamma_{p}\right)}\left(g_{p}, h_{p}\right)$. Recall that if we will identify $G_{\mathcal{F}}$ with its image under the embedding $G_{\mathcal{F}} \rightarrow \operatorname{Sym}\left(H_{\mathcal{F}}\right)$, then

$$
C_{G_{\mathcal{F}}}(\alpha, \beta)=\prod_{\mathcal{F}} \lambda_{p}\left[\Gamma_{p}\right]
$$

corresponds to the image $\lambda\left[H_{\mathcal{F}}\right]$ of the left regular permutation representation of $H_{\mathcal{F}}$; and since $\pi$ corresponds to conjugation by a suitable element of the normalizer of $G_{\mathcal{F}}$ in $\operatorname{Sym}\left(H_{\mathcal{F}}\right)$, it follows that $\prod_{\mathcal{F}} \Delta_{p}$ also corresponds to a regular subgroup of $\operatorname{Sym}\left(H_{\mathcal{F}}\right)$. This easily implies that

$$
A=\left\{p \in \mathbb{P} \mid \Delta_{p} \text { is a regular subgroup of } \operatorname{Sym}\left(\Gamma_{p}\right)\right\} \in \mathcal{F} .
$$

Furthermore, by Thomas [7], there exists a first-order sentence $\sigma$ such that if $L$ is any group, then

$$
L \vDash \sigma \quad \Longleftrightarrow \quad L \cong P S L_{2}(K) \text { for some field } K .
$$

Since $\prod_{\mathcal{F}} \Delta_{p} \cong \prod_{\mathcal{F}} \lambda_{p}\left[\Gamma_{p}\right] \cong P S L_{2}\left(\prod_{\mathcal{F}} \mathbb{F}_{p}\right)$, it follows that

$$
B=\left\{p \in \mathbb{P} \mid \Delta_{p} \cong P S L_{2}(K) \text { for some field } K\right\} \in \mathcal{F} .
$$

If $p \in A \cap B$, then $\left|\Delta_{p}\right|=\left|\Gamma_{p}\right|$ and it follows that $\Delta_{p}$ and $\lambda_{p}\left[\Gamma_{p}\right]$ are isomorphic regular subgroups of $\operatorname{Sym}\left(\Gamma_{p}\right)$, which implies that $\Delta_{p}$ is conjugate to $\lambda_{p}\left[\Gamma_{p}\right]$ inside $\operatorname{Sym}\left(\Gamma_{p}\right)$. It follows that $\prod_{\mathcal{F}} \Delta_{p}$ is conjugate to $\prod_{\mathcal{F}} \lambda_{p}\left[\Gamma_{p}\right]$ inside $G_{\mathcal{F}}$. Hence, after adjusting $\pi$ by an inner automorphism of $G_{\mathcal{F}}$ if necessary, we can suppose that

$$
\pi\left[\prod_{\mathcal{F}} \lambda_{p}\left[\Gamma_{p}\right]\right]=\prod_{\mathcal{F}} \lambda_{p}\left[\Gamma_{p}\right] .
$$


Applying Lemma 3.2, it follows that $\pi$ corresponds to conjugation by an element of $\operatorname{Hol}\left(H_{\mathcal{F}}\right)=\lambda\left[H_{\mathcal{F}}\right] \rtimes \operatorname{Aut}\left(H_{\mathcal{F}}\right)$. By Lemma 2.2,

$$
\operatorname{Aut}\left(H_{\mathcal{F}}\right)=\operatorname{Aut}\left(\prod_{\mathcal{F}} P S L_{2}\left(\mathbb{F}_{p}\right)\right)=\operatorname{Int}\left(\prod_{\mathcal{F}} P S L_{2}\left(\mathbb{F}_{p}\right)\right) ;
$$

and hence $\lambda\left[H_{\mathcal{F}}\right] \rtimes \operatorname{Aut}\left(H_{\mathcal{F}}\right)$ corresponds to a subgroup of $G_{\mathcal{F}}$. Thus $\pi$ is an inner automorphism of $G_{\mathcal{F}}$, as required.

\section{REFERENCES}

[1] C. C. Chang and J. Keisler, Model theory, North-Holland, 1990.

[2] M. Hall, The theory of groups, The Macmillan Company, New York, 1959.

[3] O. H. Kegel and B.A.F. Wehrfritz, Locally finite groups, North Holland, Amsterdam, 1973.

[4] O. Schreier and B. L. van der Waerden, Die Automorphismen der projektiven Gruppen, Abh. Math. Sem. Univ. Hamburg 6 (1928), 303-322.

[5] W. R. Scott, Group theory, Dover, New York, 1987.

[6] S. Shelah, Vive la différence III, Israel J. Math. 166 (2008), 61-96.

[7] S. Thomas, Classification theory of simple locally finite groups, Ph. D. dissertation, University of London, 1983.

Institut Für Mathematische Logik Und Grundlagenforschung, Universität MÜNSter, Einsteinstr. 62, 48149 Münster, Germany

E-mail address: philipp.luecke@uni-muenster.de

Mathematics Department, Rutgers University, 110 Frelinghuysen Road, Piscataway, New Jersey 08854-8019, USA

E-mail address: sthomas@math.rutgers.edu 